\title{
Norm Comparison Estimates for the Composite Operator
}

\author{
Xuexin Li, Yong Wang, and Yuming Xing \\ Department of Mathematics, Harbin Institute of Technology, Harbin 150001, China \\ Correspondence should be addressed to Yuming Xing; xyuming@hit.edu.cn
}

Received 12 November 2013; Revised 6 February 2014; Accepted 20 February 2014; Published 30 March 2014

Academic Editor: Shusen Ding

Copyright (C) 2014 Xuexin Li et al. This is an open access article distributed under the Creative Commons Attribution License, which permits unrestricted use, distribution, and reproduction in any medium, provided the original work is properly cited.

This paper obtains the Lipschitz and BMO norm estimates for the composite operator $\mathbb{M}_{s} \circ P$ applied to differential forms. Here, $\mathbb{M}_{s}$ is the Hardy-Littlewood maximal operator, and $P$ is the potential operator. As applications, we obtain the norm estimates for the Jacobian subdeterminant and the generalized solution of the quasilinear elliptic equation.

\section{Introduction}

Since the differential form has been proposed by Elie Cartan, its development is obvious. Differential form theory has been applied to many fields, such as partial differential equations, nonlinear analysis, and control theory. Particularly, it is an important part in differential forms to research norm inequalities for an operator or composite operator. $L^{p}$ norm has been studied very thoroughly [1-3], so we try to establish the Lipschitz norm and BMO norm inequalities for the composition of the Hardy-Littlewood maximal operator and potential operator. It is useful to explore the properties of the weighted norms; see [4-7]. So, we also research the weighted Lipschitz norm and the weighted BMO norm inequalities.

As the traditional notations, we write $\Omega$ for a bounded convex domain in $\mathbb{R}^{n}, n \geq 2$, endowed with the usual Lebesgue measure denoted by $|\Omega|$. $B$ and $\sigma B$ are concentric balls, with $\operatorname{diam}(\sigma B)=\sigma \operatorname{diam}(B)$. $\omega$ denotes a weight defined by $\omega \in L_{\text {loc }}\left(\mathbb{R}^{n}\right)$, and $\omega>0$ a.e.. The $l$-form, denoted by $\Lambda^{l}=\Lambda^{l}\left(\mathbb{R}^{n}\right)$, is a $l$-vector, spanned by exterior products $e_{I}=e_{i_{1}} \wedge e_{i_{2}} \wedge \cdots \wedge e_{i_{1}}$, for all ordered $l$-tuples $I=\left(i_{1}, i_{2}, \ldots, i_{l}\right)$, $1 \leq i_{1}<i_{2}<\cdots<i_{l} \leq n$. The $l$-form $u(x)=\Sigma_{I} u_{I}(x) d x_{I}$ is called a differential $l$-form, if $u_{I}$ is differential. We use $D^{\prime}\left(\Omega, \Lambda^{l}\right)$ to denote the differential $l$-form and $L^{s}\left(\Omega, \Lambda^{l}\right)$ to denote the $l$-form $u(x)$ on $\Omega$ satisfying $\int_{\Omega}\left|u_{I}\right|^{s}<\infty$. In particular, we know that the 0 -form is a function. We define the exterior derivative $d: D^{\prime}\left(\Omega, \Lambda^{l}\right) \rightarrow D^{\prime}\left(\Omega, \Lambda^{l+1}\right)$ by

$$
\begin{aligned}
& d(u(x)) \\
& =\sum_{k=1}^{n} \sum_{1 \leq i_{1}<i_{2}<\cdots<i_{l} \leq n} \frac{\partial u_{i_{1} i_{2} \cdots i_{l}}(x)}{\partial x_{k}} \\
& \times d x_{k} \wedge d x_{i_{1}} \wedge \cdots \wedge d x_{i_{l}}, \\
& l=0,1, \ldots, n-1 .
\end{aligned}
$$

We define $\star$ by

$$
\star \mathcal{U}=\operatorname{sign}(\pi) u_{i_{1} i_{2} \cdots i_{l}}(x) d x_{j_{1}} \wedge \cdots \wedge d x_{j_{n-l}},
$$

where $\pi=\left(i_{1}, \ldots, i_{l}, j_{1}, \ldots, j_{n-l}\right)$ is a permutation of $(1, \ldots, n)$ and $\operatorname{sign}(\pi)$ is the signature of the permutation. Now, we can define the Hodge codifferential $d^{\star}$ like this

$$
\begin{array}{r}
d^{\star}=(-1)^{n l+1} \star d \star: D^{\prime}\left(\Omega, \Lambda^{l+1}\right) \\
\longrightarrow=0,1, \ldots, n-1 .
\end{array}
$$

The differential form we research here satisfies the nonhomogeneous $A$-harmonic equation

$$
d^{\star} A(x, d u)=B(x, d u),
$$


where $A: \Omega \times \Lambda^{l}\left(\mathbb{R}^{n}\right) \rightarrow \Lambda^{l}\left(\mathbb{R}^{n}\right)$ and $B: \Omega \times \Lambda^{l}\left(\mathbb{R}^{n}\right) \rightarrow$ $\Lambda^{l-1}\left(\mathbb{R}^{n}\right)$ satisfy the conditions

$$
\begin{gathered}
|A(x, \xi)| \leq a|\xi|^{p-1}, \\
A(x, \xi) \cdot \xi \geq|\xi|^{p}, \quad|B(x, \xi)| \leq b|\xi|^{p-1}
\end{gathered}
$$

for almost every $x \in \Omega$ and all $\xi \in \wedge^{l}\left(\mathbb{R}^{n}\right)$. Here $a, b>0$ are constants and $1<p<\infty$ is a fixed exponent associated with (4).

\section{Lipschitz and BMO Norm Inequalities}

In this section, we introduce some definitions. Then, we give main lemmas used in theorems. Finally, we give the norm comparison estimates for the composite operator.

For $u \in L_{\text {loc }}^{1}\left(\Omega, \Lambda^{l}\right), l=0,1, \ldots, n$, we write $u \in$ loc $\operatorname{Lip}_{k}\left(\Omega, \Lambda^{l}\right), 0 \leq k \leq 1$, if

$$
\|u\|_{\text {loc } \operatorname{Lip}_{k}, \Omega}=\sup _{\sigma \mathrm{Q} \subset \Omega}|Q|^{-(n+k) / n}\left\|u-u_{\mathrm{Q}}\right\|_{1, \mathrm{Q}}<\infty
$$

for some $\sigma>1$.

For $u \in L_{\text {loc }}^{1}\left(\Omega, \Lambda^{l}\right), l=0,1, \ldots, n$, we say $u \in$ $\operatorname{BMO}\left(\Omega, \Lambda^{l}\right)$, if

$$
\|u\|_{*, \Omega}=\sup _{\sigma \mathrm{Q} \subset \Omega}|Q|^{-1}\left\|u-u_{\mathrm{Q}}\right\|_{1, \mathrm{Q}}<\infty
$$

for some $\sigma>1$.

Hardy-Littlewood maximal operator is defined by

$$
\mathbb{M}_{s}(u)=\mathbb{M}_{s} u(x)=\sup _{r>0}\left(\frac{1}{|B(x, r)|} \int_{B(x, r)}|u(y)|^{s} d y\right)^{1 / s},
$$

where $1 \leq s<\infty, u$ is a locally $L^{s}$-integrable form and $B(x, r)$ is a ball with radius $r$.

Potential operator is first extended to differential form by $\mathrm{Bi}$ in [8], which is defined as follows:

$$
P u=P u(x)=\sum_{I} \int_{\Omega} K(x, y) u_{I}(y) d y d x_{I},
$$

where the kernel $K(x, y)$ is a nonnegative measurable function defined for $x \neq y, u$ is a differential $l$-form, and the summation is over all ordered $l$-tuples $I$.

We need the following two lemmas for the HardyLittlewood maximal operator and potential operator to prove Theorem 7.

Lemma 1 (see [9]). Let $u \in L^{t}\left(\Omega, \Lambda^{l}\right), l=0,1, \ldots, n$, be a differential form in a domain $\Omega$ and $\mathbb{M}_{s}$ be the HardyLittlewood maximal operator, $1 \leq s<t<\infty$. Then, $\mathbb{M}_{s}(u) \in$ $L^{t}(\Omega)$ and there exists a constant $C$, independent of $u$, such that

$$
\left\|\mathbb{M}_{s}(u)\right\|_{t, \Omega} \leq C\|u\|_{t, \Omega} .
$$

Lemma 2 (see [8]). Let $u \in D^{\prime}\left(\Omega, \Lambda^{l}\right), l=0,1, \ldots, n$, be a differential form in a domain $\Omega$ and let $P$ be the potential operator. Then, there exists a constant $C$, independent of $u$, such that

$$
\|P(u)\|_{s, \Omega} \leq C\|u\|_{s, \Omega},
$$

where $1<s<\infty$.

The following lemma was established by Iwaniec and Lutoborski in [10].

Lemma 3. Let $u \in L^{s}\left(\Omega, \Lambda^{l}\right), l=0,1, \ldots, n, 1<s<\infty$, be a differential form in a domain $\Omega$. Then, there exists a constant $C$, independent of $u$, such that

$$
\left\|u_{\Omega}\right\|_{s, \Omega} \leq C\|u\|_{s, \Omega},
$$

where $u_{\Omega}$ is a closed form.

We will use the following generalized Hölder inequality repeatedly in this paper.

Lemma 4. Let $0<q<\infty, 0<p<\infty$, and $s^{-1}=q^{-1}+p^{-1}$. If $f$ and $g$ are measurable functions on $\mathbb{R}^{n}$, then

$$
\|f g\|_{s, \Omega} \leq\|f\|_{q, \Omega}\|g\|_{p, \Omega}
$$

for any $\Omega \subset \mathbb{R}^{n}$.

We also need the Caccioppoli inequality for differential form.

Lemma 5 (see [11]). Let $u \in D^{\prime}\left(\Omega, \Lambda^{l}\right), l=0,1, \ldots, n$, be a solution of the nonhomogeneous A-harmonic equation (4) in $\Omega$. Then, there exists a constant $C$, independent of $u$, such that

$$
\|d u\|_{s, B} \leq C \operatorname{diam}(B)^{-1}\|u-c\|_{s, \sigma B}
$$

for all balls with $\sigma B \subset \Omega$ and any closed form $c$, where $1<s<$ $\infty$, and $\sigma>1$.

The following Poincaré inequality appears in [5].

Lemma 6. Let $u \in D^{\prime}\left(\Omega, \Lambda^{l}\right), l=0,1, \ldots, n-1$, be a solution of the nonhomogeneous A-harmonic equation (4) in $\Omega$. Then, there exists a constant $C$, independent of $u$, such that

$$
\left\|u-u_{B}\right\|_{s, B} \leq C|B| \operatorname{diam}(B)\|d u\|_{s, B}
$$

for any ball B in $\Omega$.

First, we establish a Poincaré-type inequality for the composite operator $\mathbb{M}_{s} \circ P$.

Theorem 7. Let $u \in L^{t}\left(\Omega, \Lambda^{l}\right), l=1,2, \ldots, n, 1 \leq s<t<$ $\infty$, be a differential form in a domain $\Omega$. Then, there exists a constant $C$, independent of $u$, such that

$$
\left\|\mathbb{M}_{s} P(u)-\left(\mathbb{M}_{s} P(u)\right)_{\Omega}\right\|_{t, \Omega} \leq C\|u\|_{t, \Omega} .
$$


Proof. From Lemma 3 and Minkowski inequality, we have

$$
\begin{aligned}
\left\|u-u_{\Omega}\right\|_{t, \Omega} & =\left(\int_{\Omega}\left|u(x)-u_{\Omega}(x)\right|^{t} d x\right)^{1 / t} \\
& \leq\left(\int_{\Omega}|u(x)|^{t} d x\right)^{1 / t}+\left(\int_{\Omega}\left|u_{\Omega}(x)\right|^{t} d x\right)^{1 / t} \\
& \leq\left(\int_{\Omega}|u(x)|^{t} d x\right)^{1 / t}+C_{1}\left(\int_{\Omega}|u(x)|^{t} d x\right)^{1 / t} \\
& \leq C_{2}\|u\|_{t, \Omega} .
\end{aligned}
$$

Replacing $u$ with $\mathbb{M}_{s} P(u)$ and combining Lemmas 1 and 2, we obtain

$$
\begin{aligned}
\left\|\mathbb{M}_{s} P(u)-\left(\mathbb{M}_{s} P(u)\right)_{\Omega}\right\|_{t, \Omega} & \leq C_{2}\left\|\mathbb{M}_{s} P(u)\right\|_{t, \Omega} \\
& \leq C_{3}\|P(u)\|_{t, \Omega} \\
& \leq C_{4}\|u\|_{t, \Omega} .
\end{aligned}
$$

Theorem 7 has been completed.

Then, we estimate the Lipschitz norm of $\mathbb{M}_{s} P(u)$ in terms of $L^{t}$-norm.

Theorem 8. Let $u \in L^{t}\left(\Omega, \Lambda^{l}\right), l=1,2, \ldots, n, 1 \leq s<t<\infty$, be a solution of the nonhomogeneous A-harmonic equation (4) in $\Omega$. Then, there exists a constant $C$, independent of $u$, such that

$$
\left\|\mathbb{M}_{s} P(u)\right\|_{\operatorname{loc~Lip}_{k}, \Omega} \leq C\left\|d \mathbb{M}_{s} P(u)\right\|_{t, \Omega},
$$

where $k$ is a constant with $0 \leq k \leq 1$.

Proof. Setting $u=\mathbb{M}_{s} P(u)$ in Lemma 6 we have

$$
\left\|\mathbb{M}_{s} P(u)-\left(\mathbb{M}_{s} P(u)\right)_{B}\right\|_{t, B} \leq C_{1}|B| \operatorname{diam}(B)\left\|d \mathbb{M}_{s} P(u)\right\|_{t, B}
$$

for all balls $B$ with $B \subset \Omega$. Using Hölder inequality, we have

$$
\begin{array}{rl}
\| \mathbb{M}_{s} & P(u)-\left(\mathbb{M}_{s} P(u)\right)_{B} \|_{1, B} \\
\leq & \left(\int_{B}\left|\mathbb{M}_{s} P(u)-\left(\mathbb{M}_{s} P(u)\right)_{B}\right|^{t} d x\right)^{1 / t} \\
& \times\left(\int_{B} 1^{t /(t-1)} d x\right)^{(t-1) / t} \\
\leq & |B|^{(t-1) / t}\left\|\mathbb{M}_{s} P(u)-\left(\mathbb{M}_{s} P(u)\right)_{B}\right\|_{t, B} \\
= & |B|^{1-1 / t}\left\|\mathbb{M}_{s} P(u)-\left(\mathbb{M}_{s} P(u)\right)_{B}\right\|_{t, B} \\
\leq & |B|^{1-1 / t} C_{1}|B| \operatorname{diam}(B)\left\|d \mathbb{M}_{s} P(u)\right\|_{t, B} \\
\leq & C_{2}|B|^{2-1 / t+1 / n}\left\|d \mathbb{M}_{s} P(u)\right\|_{t, B} .
\end{array}
$$

From the definition of Lipschitz norm and (21), it follows that

$$
\begin{aligned}
& \left\|\mathbb{M}_{s} P(u)\right\|_{\text {loc } \operatorname{Lip}_{k}, \Omega} \\
& =\sup _{\sigma B \subset \Omega}|B|^{-(n+k) / n}\left\|\mathbb{M}_{s} P(u)-\left(\mathbb{M}_{s} P(u)\right)_{B}\right\|_{1, B} \\
& =\sup _{\sigma B \subset \Omega}|B|^{-1-k / n}\left\|\mathbb{M}_{s} P(u)-\left(\mathbb{M}_{s} P(u)\right)_{B}\right\|_{1, B} \\
& \leq \sup _{\sigma B \subset \Omega}|B|^{-1-k / n} C_{2}|B|^{2-1 / t+1 / n}\left\|d \mathbb{M}_{s} P(u)\right\|_{t, B} \\
& =\sup _{\sigma B \subset \Omega} C_{2}|B|^{1-k / n-1 / t+1 / n}\left\|d \mathbb{M}_{s} P(u)\right\|_{t, B} \\
& \leq \sup _{\sigma B \subset \Omega} C_{2}|\Omega|^{1-k / n-1 / t+1 / n}\left\|d \mathbb{M}_{s} P(u)\right\|_{t, B} \\
& \leq C_{3} \sup _{\sigma B \subset \Omega}\left\|d \mathbb{M}_{s} P(u)\right\|_{t, B} \\
& \leq C_{3}\left\|d \mathbb{M}_{s} P(u)\right\|_{t, \Omega} .
\end{aligned}
$$

Theorem 8 has been completed.

Now, we establish the norm comparison theorems of the composite operator between Lipschitz norm and BMO norm.

Theorem 9. Let $u \in L^{s}\left(\Omega, \Lambda^{l}\right), l=1,2, \ldots, n, 1 \leq s<\infty$, be a differential form in $\Omega$. Then, there exists a constant $C$, independent of $u$, such that

$$
\left\|\mathbb{M}_{s} P(u)\right\|_{*, \Omega} \leq C\left\|\mathbb{M}_{s} P(u)\right\|_{\operatorname{loc}^{2} \operatorname{Lip}_{k}, \Omega},
$$

where $k$ is a constant with $0 \leq k \leq 1$.

Proof. From the definition of BMO norm, we have

$$
\begin{aligned}
& \left\|\mathbb{M}_{s} P(u)\right\|_{*, \Omega} \\
& =\sup _{\sigma B \subset \Omega}|B|^{-1}\left\|\mathbb{M}_{s} P(u)-\left(\mathbb{M}_{s} P(u)\right)_{B}\right\|_{1, B} \\
& =\sup _{\sigma B \subset \Omega}|B|^{k / n}|B|^{-(n+k) / n}\left\|\mathbb{M}_{s} P(u)-\left(\mathbb{M}_{s} P(u)\right)_{B}\right\|_{1, B} \\
& \leq \sup _{\sigma B \subset \Omega}|\Omega|^{k / n}|B|^{-(n+k) / n}\left\|\mathbb{M}_{s} P(u)-\left(\mathbb{M}_{s} P(u)\right)_{B}\right\|_{1, B} \\
& \leq|\Omega|^{k / n} \sup _{\sigma B \subset \Omega}|B|^{-(n+k) / n}\left\|\mathbb{M}_{s} P(u)-\left(\mathbb{M}_{s} P(u)\right)_{B}\right\|_{1, B} \\
& \leq C \sup _{\sigma B \subset \Omega}|B|^{-(n+k) / n}\left\|\mathbb{M}_{s} P(u)-\left(\mathbb{M}_{s} P(u)\right)_{B}\right\|_{1, B} \\
& \leq C\left\|\mathbb{M}_{s} P(u)\right\|_{l o c} \operatorname{Lip}_{k}, \Omega .
\end{aligned}
$$

Theorem 9 has been completed.

Theorem 10. Let $u \in L^{s}\left(\Omega, \Lambda^{l}\right), l=1,2, \ldots, n, 1<s<\infty$, be a solution of the nonhomogeneous A-harmonic equation (4) in $\Omega$. Then, there exists a constant $C$, independent of $u$, such that

$$
\left\|\mathbb{M}_{s} P(u)\right\|_{\operatorname{loc} \operatorname{Lip}_{k}, \Omega} \leq C\left\|\mathbb{M}_{s} P(u)\right\|_{*, \Omega},
$$

where $k$ is a constant with $0 \leq k \leq 1$. 
Proof. From (21), we obtain

$$
\begin{aligned}
& \left\|\mathbb{M}_{s} P(u)-\left(\mathbb{M}_{s} P(u)\right)_{B}\right\|_{1, B} \\
& \quad \leq C_{1}|B|^{2-1 / s+1 / n}\left\|d \mathbb{M}_{s} P(u)\right\|_{s, B} .
\end{aligned}
$$

Based on Lemma 5, we get

$$
\left\|d \mathbb{M}_{s} P(u)\right\|_{s, B} \leq C_{2} \operatorname{diam}(B)^{-1}\left\|\mathbb{M}_{s} P(u)-\left(\mathbb{M}_{s} P(u)\right)_{B}\right\|_{s, \sigma B} .
$$

From the weak reverse Hölder inequality, it follows that

$$
\begin{aligned}
& \left\|\mathbb{M}_{s} P(u)-\left(\mathbb{M}_{s} P(u)\right)_{B}\right\|_{s, \sigma B} \\
& \leq C_{3}|B|^{(1-s) / s}\left\|\mathbb{M}_{s} P(u)-\left(\mathbb{M}_{s} P(u)\right)_{B}\right\|_{1, \sigma^{\prime} B},
\end{aligned}
$$

where $\sigma^{\prime}>\sigma>1$. So, we get

$$
\begin{aligned}
& \left\|\mathbb{M}_{s} P(u)-\left(\mathbb{M}_{s} P(u)\right)_{B}\right\|_{1, B} \\
& \quad \leq C_{1}|B|^{2-1 / s+1 / n}\left\|d \mathbb{M}_{s} P(u)\right\|_{s, B} \\
& \quad \leq C_{4}|B|\left\|\mathbb{M}_{s} P(u)-\left(\mathbb{M}_{s} P(u)\right)_{B}\right\|_{1, \sigma^{\prime} B} .
\end{aligned}
$$

Let $\sigma^{\prime \prime}>\sigma^{\prime}$; we obtain

$$
\begin{aligned}
& \left\|\mathbb{M}_{s} P(u)\right\|_{10 c \operatorname{Lip}_{k}, \Omega} \\
& =\sup _{\sigma^{\prime \prime} B \subset \Omega}|B|^{-(n+k) / n}\left\|\mathbb{M}_{s} P(u)-\left(\mathbb{M}_{s} P(u)\right)_{B}\right\|_{1, B} \\
& \leq \sup _{\sigma^{\prime \prime} B \subset \Omega} C_{4}|B|^{1-(k / n)}|B|^{-1}\left\|\mathbb{M}_{s} P(u)-\left(\mathbb{M}_{s} P(u)\right)_{B}\right\|_{1, \sigma^{\prime} B} \\
& \leq C_{5} \sup _{\sigma^{\prime \prime} B \subset \Omega} C_{4}|B|^{-1}\left\|\mathbb{M}_{s} P(u)-\left(\mathbb{M}_{s} P(u)\right)_{B}\right\|_{1, \sigma^{\prime} B} \\
& \leq C_{6}\left\|\mathbb{M}_{s} P(u)\right\|_{*, \Omega} .
\end{aligned}
$$

Theorem 10 has been completed.

Combining Theorems 8 and 9, we obtain the following result easily.

Corollary 11. Let $u \in L^{t}\left(\Omega, \Lambda^{l}\right), l=1,2, \ldots, n, 1 \leq s<t<$ $\infty$, be a solution of the nonhomogeneous A-harmonic equation (4) in $\Omega$. Then, there exists a constant $C$, independent of $u$, such that

$$
\left\|\mathrm{M}_{s} P(u)\right\|_{*, \Omega} \leq C\left\|d \mathbb{M}_{s} P(u)\right\|_{t, \Omega} .
$$

\section{The Weighted Lipschitz and BMO Norm Inequalities}

In this section, we obtain some weighted inequalities for the composition operator $\mathbb{M}_{s} \circ P$.

The weight function we use here is $A(\alpha, \beta, \gamma, \Omega)$ weight. The $A(\alpha, \beta, \gamma, \Omega)$-class is a new weight class which was first proposed by Xing in [7]. It contains the well-known $A_{r}(\Omega)$ weight as a proper subset.
Definition 12. One says that a measurable function $\omega(x)$ defined on a subset $\Omega \subset \mathbb{R}^{n}$ satisfies the $A(\alpha, \beta, \gamma, \Omega)$ condition for some positive constants $\alpha, \beta, \gamma$, if $\omega(x)>0$ a.e., and

$$
\sup _{B \subset \Omega}\left(\frac{1}{|B|} \int_{B} \omega^{\alpha} d x\right)\left(\frac{1}{|B|} \int_{B} \omega^{-\beta} d x\right)^{\gamma / \beta}<\infty
$$

Let $u \in L_{\text {loc }}^{1}\left(\Omega, \Lambda^{l}, \omega\right), l=0,1,2, \ldots, n$. We say $u \in$ $\operatorname{loc} \operatorname{Lip}_{k}\left(\Omega, \Lambda^{l}, \omega\right), 0 \leq k \leq 1$, if

$$
\|u\|_{\text {loc } \operatorname{Lip}_{k}, \Omega, \omega}=\sup _{\sigma \mathrm{Q} \subset \Omega}(\mu(Q))^{-(n+k) / n}\left\|u-u_{\mathrm{Q}}\right\|_{1, \mathrm{Q}, \omega}<\infty
$$

for some $\sigma>1$, where $\Omega$ is bounded in $\mathbb{R}^{n}, \omega$ is a weight, and $\mu$ is Radon measure defined by $d \mu=\omega(x) d x$.

Let $u \in L_{\text {loc }}^{1}\left(\Omega, \Lambda^{l}, \omega\right), l=0,1,2, \ldots, n$. We say $u \in$ $\operatorname{BMO}\left(\Omega, \Lambda^{l}, \omega\right)$, if

$$
\|u\|_{*, \Omega, \omega}=\sup _{\sigma \mathrm{Q} \subset \Omega}(\mu(\mathrm{Q}))^{-1}\left\|u-u_{\mathrm{Q}}\right\|_{1, \mathrm{Q}, \omega}<\infty
$$

for some $\sigma>1$, where $\Omega$ is bounded in $\mathbb{R}^{n}, \omega$ is a weight, and $\mu$ is Radon measure defined by $d \mu=\omega(x) d x$.

Now, we estimate the weighted Lipschitz and BMO norm for the composition operator $\mathbb{M}_{s} \circ P$.

Theorem 13. Let $u \in L^{q}\left(\Omega, \Lambda^{l}, \mu\right), l=1,2, \ldots, n, 1 \leq s<q<$ $\infty$, be a solution of the nonhomogeneous $A$-harmonic equation (4) in $\Omega$. Radon measure $\mu$ is defined by $\omega(x) d x=d \mu$, and $\omega(x) \in A(\alpha, \beta, \gamma, \Omega)$ for some $\alpha>1$, where $1<p<\infty$, $\beta=\alpha q /(\alpha p-p-\alpha q), \gamma=\alpha q / p$, and $\alpha p-p-\alpha q>0$. Then, there exists a constant $C$, independent of $u$, such that

$$
\left\|\mathbb{M}_{s} P(u)\right\|_{\operatorname{loc} \operatorname{Lip}_{k}, \Omega, \omega} \leq C\left\|d \mathbb{M}_{s} P(u)\right\|_{p, \Omega, \omega} .
$$

Proof. Using Hölder inequality with $1 / q=1 / \alpha q+(\alpha-1) / \alpha q$, we have

$$
\begin{aligned}
& \left(\int_{B}\left|\mathbb{M}_{s} P(u)-\left(\mathbb{M}_{s} P(u)\right)_{B}\right|^{q} \omega(x) d x\right)^{1 / q} \\
& =\left(\int_{B}\left|\mathbb{M}_{s} P(u)-\left(\mathbb{M}_{s} P(u)\right)_{B} \omega(x)^{1 / q}\right|^{q} d x\right)^{1 / q} \\
& \leq\left(\int_{B}\left|\mathbb{M}_{s} P(u)-\left(\mathbb{M}_{s} P(u)\right)_{B}\right|^{\alpha q /(\alpha-1)} d x\right)^{(\alpha-1) / \alpha q} \\
& \quad \times\left(\int_{B}\left(\omega(x)^{1 / q}\right)^{\alpha q} d x\right)^{1 / \alpha q} \\
& \leq C_{1}|B| \operatorname{diam}(B)\left(\int_{B}\left|d \mathbb{M}_{s} P(u)\right|^{\alpha q /(\alpha-1)} d x\right)^{(\alpha-1) / \alpha q} \\
& \quad \times\left(\int_{B} \omega(x)^{\alpha} d x\right)^{1 / \alpha q} .
\end{aligned}
$$


Using Hölder inequality with $(\alpha-1) / \alpha q=(1 / p)+(\alpha p-p-$ $\alpha q) / \alpha q p$, we obtain

$$
\begin{aligned}
& \left(\int_{B}\left|d \mathbb{M}_{s} P(u)\right|^{\alpha q /(\alpha-1)} d x\right)^{(\alpha-1) / \alpha q} \\
& =\left(\int_{B}\left|d \mathbb{M}_{S} P(u) \omega(x)^{1 / p} \omega(x)^{-1 / p}\right|^{\alpha q /(\alpha-1)} d x\right)^{(\alpha-1) / \alpha q} \\
& \leq\left(\int_{B}\left|d \mathbb{M}_{S} P(u) \omega(x)^{1 / p}\right|^{p} d x\right)^{1 / p} \\
& \quad \times\left(\int_{B}\left(\omega(x)^{-1 / p}\right)^{\alpha q p /(\alpha p-p-\alpha q)} d x\right)^{(\alpha p-p-\alpha q) / \alpha q p} \\
& =\left(\int_{B}\left|d \mathbb{M}_{S} P(u)\right|^{p} \omega(x) d x\right)^{1 / p} \\
& \quad \times\left(\int_{B}\left(\frac{1}{\omega(x)}\right)^{\alpha q /(\alpha p-p-\alpha q)} d x\right)^{(\alpha p-p-\alpha q) / \alpha q p}
\end{aligned}
$$

Since $\omega(x) \in A(\alpha, \alpha q /(\alpha p-p-\alpha q), \alpha q / p, \Omega)$, we get

$$
\begin{gathered}
\left(\int_{B} \omega(x)^{\alpha} d x\right)^{1 / \alpha q}\left(\int_{B}\left(\frac{1}{\omega(x)}\right)^{\alpha q /(\alpha p-p-\alpha q)} d x\right)^{(\alpha p-p-\alpha q) / \alpha q p} \\
=\left(\left(\int_{B} \omega(x)^{\alpha} d x\right)\right. \\
\left.\quad \times\left(\int_{B}\left(\frac{1}{\omega(x)}\right)^{\alpha q /(\alpha p-p-\alpha q)} d x\right)^{(\alpha p-p-\alpha q) / p}\right)^{1 / \alpha q} \\
=|B|^{2 / \alpha q} \\
\times\left(\left(\frac{1}{|B|}(\omega(x))^{\alpha} d x\right)\right. \\
\left.\quad \times\left(\frac{1}{|B|} d x\right)^{(\alpha p-p-\alpha q) / p}\right)^{1 / \alpha q}
\end{gathered}
$$$$
\leq C_{2} \text {. }
$$

From (36), (37), and (38), we know that

$$
\begin{aligned}
& \left(\int_{B}\left|\mathbb{M}_{S} P(u)-\left(\mathbb{M}_{s} P(u)\right)_{B}\right|^{q} \omega(x) d x\right)^{1 / q} \\
& \quad \leq C_{3}|B| \operatorname{diam}(B)\left(\int_{B}\left|d \mathbb{M}_{s} P(u)\right|^{p} \omega(x) d x\right)^{1 / p} .
\end{aligned}
$$

So, we obtain

$$
\begin{aligned}
&\left\|\mathbb{M}_{s} P(u)-\left(\mathbb{M}_{s} P(u)\right)_{B}\right\|_{1, B, \omega} \\
&=\int_{B}\left|\mathbb{M}_{s} P(u)-\left(\mathbb{M}_{s} P(u)\right)_{B}\right| d \mu \\
& \leq\left(\int_{B}\left|\mathbb{M}_{s} P(u)-\left(\mathbb{M}_{s} P(u)\right)_{B}\right|^{q} \omega(x) d x\right)^{1 / q} \\
& \times\left(\int_{B} 1^{q /(q-1)} d \mu\right)^{(q-1) / q} \\
&=(\mu(B))^{(q-1) / q}\left\|\mathbb{M}_{s} P(u)-\left(\mathbb{M}_{s} P(u)\right)_{B}\right\|_{q, B, \omega} \\
& \leq C_{4}(\mu(B))^{(q-1) / q}|B| \operatorname{diam}(B)\left\|d \mathbb{M}_{s} P(u)\right\|_{P, B, \omega} .
\end{aligned}
$$

Based on the definition of the weighted Lipschitz norm and (40), we have

$$
\begin{aligned}
& \left\|\mathbb{M}_{s} P(u)\right\|_{10 c} \operatorname{Lip}_{k}, \Omega, \omega \\
& =\sup _{\sigma B \subset \Omega}(\mu(B))^{-(n+k) / n}\left\|\mathbb{M}_{s} P(u)-\left(\mathbb{M}_{s} P(u)\right)_{B}\right\|_{1, B, \omega} \\
& \leq C_{5} \sup _{\sigma B \subset \Omega}(\mu(B))^{-(n+k) / n+(q-1) / q+1+1 / n} \\
& \quad \times\left\|d \mathbb{M}_{s} P(u)\right\|_{p, B, \omega} \\
& \leq C_{5} \sup (\mu(\Omega))^{-(n+k) / n+(q-1) / q+1+1 / n} \\
& \quad \times\left\|d \mathbb{M}_{s} P(u)\right\|_{p, B, \omega} \\
& \leq C\left\|d \mathbb{M}_{s} P(u)\right\|_{p, \Omega, \omega} .
\end{aligned}
$$

Theorem 13 has been completed.

Theorem 14. Let $u \in L^{s}\left(\Omega, \Lambda^{l}, \mu\right), l=1,2, \ldots, n, 1 \leq s<$ $\infty$, be a differential form in $\Omega$. Radon measure $\mu$ is defined by $\omega(x) d x=d \mu$, and $\omega(x) \in A(\alpha, \beta, \gamma, \Omega)$. Then, there exists $a$ constant $C$, independent of $u$, such that

$$
\left\|\mathbb{M}_{s} P(u)\right\|_{*, \Omega, \omega} \leq C\left\|\mathbb{M}_{s} P(u)\right\|_{\text {loc Lip }_{k}, \Omega, \omega},
$$

where $\alpha, \beta, \gamma$ are some positive constants.

Proof. From the definition of the weighted BMO norm, we have

$$
\begin{aligned}
& \left\|\mathbb{M}_{s} P(u)\right\|_{*, \Omega, \omega} \\
& =\sup _{\sigma B \subset \Omega}(\mu(B))^{-1}\left\|\mathbb{M}_{s} P(u)-\left(\mathbb{M}_{s} P(u)\right)_{B}\right\|_{1, B, \omega} \\
& =\sup _{\sigma B \subset \Omega}(\mu(B))^{k / n}(\mu(B))^{-(n+k) / n} \\
& \quad \times\left\|\mathbb{M}_{s} P(u)-\left(\mathbb{M}_{s} P(u)\right)_{B}\right\|_{1, B, \omega} \\
& \leq \sup _{\sigma B \subset \Omega}(\mu(\Omega))^{k / n}(\mu(B))^{-(n+k) / n} \\
& \quad \times\left\|\mathbb{M}_{s} P(u)-\left(\mathbb{M}_{s} P(u)\right)_{B}\right\|_{1, B, \omega}
\end{aligned}
$$




$$
\begin{aligned}
\leq & (\mu(\Omega))^{k / n} \sup _{\sigma B \subset \Omega}(\mu(B))^{-(n+k) / n} \\
& \times\left\|\mathbb{M}_{s} P(u)-\left(\mathbb{M}_{s} P(u)\right)_{B}\right\|_{1, B, \omega} \\
\leq & C \sup _{\sigma B C \Omega}(\mu(B))^{-(n+k) / n} \\
& \times\left\|\mathbb{M}_{s} P(u)-\left(\mathbb{M}_{s} P(u)\right)_{B}\right\|_{1, B, \omega} \\
\leq & C\left\|\mathbb{M}_{s} P(u)\right\|_{\text {loc } \operatorname{Lip}_{k}, \Omega, \omega}
\end{aligned}
$$

Theorem 14 has been completed.

Combining Theorems 13 and 14, we obtain the following corollary.

Corollary 15. Let $u \in L^{q}\left(\Omega, \Lambda^{l}, \mu\right), l=1,2, \ldots, n, 1 \leq s<q<$ $\infty$, be a solution of the nonhomogeneous A-harmonic equation (4) in $\Omega$. Radon measure $\mu$ is defined by $\omega(x) d x=d \mu$, and $\omega(x) \in A(\alpha, \beta, \gamma, \Omega)$ for some $\alpha>1$, where $1<p<\infty$, $\beta=\alpha q /(\alpha p-p-\alpha q), \gamma=\alpha q / p$, and $\alpha p-p-\alpha q>0$. Then, there exists a constant $C$, independent of $u$, such that

$$
\left\|\mathbb{M}_{s} P(u)\right\|_{*, \Omega, \omega} \leq C\left\|d \mathbb{M}_{s} P(u)\right\|_{p, \Omega, \omega} .
$$

\section{Applications}

In this section, we use the theorems we obtain to estimate the norms of Jacobian subdeterminant and the generalized solution of the quasilinear elliptic equation.

Example 16. Let $u=\left(u^{1}, \ldots, u^{n}\right)$ be a map from $\Omega$ to $\mathbb{R}^{n} . J(x, u)$ is the Jacobian determinant. Now choosing the subdeterminant of the Jacobian determinant,

$$
J\left(x_{j_{1}}, x_{j_{2}} ; u^{i_{1}}, u^{i_{2}}\right)=\left|\begin{array}{ll}
\frac{\partial u^{i_{1}}}{\partial x_{j_{1}}} & \frac{\partial u^{i_{1}}}{\partial x_{j_{2}}} \\
\frac{\partial u^{i_{2}}}{\partial x_{j_{1}}} & \frac{\partial u^{i_{2}}}{\partial x_{j_{2}}}
\end{array}\right| .
$$

We know that $J\left(x_{j_{1}}, x_{j_{2}} ; u^{i_{1}}, u^{i_{2}}\right) d x_{j_{1}} \wedge d x_{j_{2}}$ is a 2 -form. Let $v=J\left(x_{j_{1}}, x_{j_{2}} ; u^{i_{1}}, u^{i_{2}}\right) d x_{j_{1}} \wedge d x_{j_{2}}$. If $v \in L^{s}\left(\Omega, \Lambda^{2}\right), 1 \leq s<\infty$, from Theorems 9 and 14, we obtain the following results:

$$
\begin{gathered}
\left\|\mathbb{M}_{s} P(v)\right\|_{*, \Omega} \leq C\left\|\mathbb{M}_{\mathcal{S}} P(v)\right\|_{\text {loc } \operatorname{Lip}_{k}, \Omega}, \\
\left\|\mathbb{M}_{s} P(v)\right\|_{*, \Omega, \omega} \leq C\left\|\mathbb{M}_{s} P(v)\right\|_{{\operatorname{loc~} \operatorname{Lip}_{k}, \Omega, \omega}}
\end{gathered}
$$

where $C$ is a constant, $0 \leq k \leq 1, \mathbb{M}_{s}$ is the Hardy-Littlewood maximal operator, $P$ is the potential operator, and $\omega$ is a $A(\alpha, \beta, \gamma, \Omega)$ weight satisfying $\alpha>1, \beta=\alpha q /(\alpha p-p-\alpha q)$, $\gamma=\alpha q / p$, and $\alpha p-p-\alpha q>0$.

Example 17. Let $u(x)=\left(u^{1}, u^{2}, \ldots, u^{n}\right): \Omega \rightarrow \mathbb{R}^{n}$ be a $K$ quasiregular mapping, $K \geq 1$. Then,

$$
v=u^{i}(x) \quad(i=1,2, \ldots, n)
$$

or

$$
v=\log |u(x)|
$$

is a generalized solution of the following equation:

$$
\operatorname{div} A(x, \nabla v)=0, \quad A=\left(A^{1}, A^{2}, \ldots, A^{n}\right) .
$$

Here, $A^{i}(x, \eta)$ can be expressed as

$$
A^{i}(x, \eta)=\frac{\partial}{\partial \eta_{i}}\left(\sum_{i, j=1}^{n} \alpha_{i, j}(x) \eta_{i} \eta_{j}\right)^{n / 2} .
$$

$\alpha_{i, j}$ in the formula above are some functions, which can be expressed in terms of the differential matrix $D u(x)$, and satisfy

$$
C_{1}(K)|\eta|^{2} \leq \sum_{i, j=1}^{n} \alpha_{i, j} \eta_{i} \eta_{j} \leq C_{2}(K)|\eta|^{2}
$$

where $C_{1}(K), C_{2}(K)>0$. If we assume that $\mathbb{M}_{s}$ is the HardyLittlewood maximal operator, $P$ is the potential operator, $0 \leq$ $k \leq 1$, and $\omega$ is a $A(\alpha, \beta, \gamma, \Omega)$ weight satisfying $\alpha>1$, $\beta=\alpha q /(\alpha p-p-\alpha q), \gamma=\alpha q / p$, and $\alpha p-p-\alpha q>$ 0 , according to Theorem 13 and Corollary 15, we obtain the following inequalities:

$$
\begin{gathered}
\left\|\mathbb{M}_{s} P(v)\right\|_{\text {loc } \operatorname{Lip}_{k}, \Omega, \omega} \leq C\left\|d \mathbb{M}_{s} P(v)\right\|_{p, \Omega, \omega}, \\
\left\|\mathbb{M}_{s} P(v)\right\|_{*, \Omega, \omega} \leq C\left\|\mathbb{M}_{s} P(v)\right\|_{p, \Omega, \omega},
\end{gathered}
$$

where $C$ is a constant.

\section{Conflict of Interests}

The authors declare that there is no conflict of interests regarding the publication of this paper.

\section{References}

[1] C. Scott, " $L^{p}$-theory of differential forms on manifolds," Transactions of the American Mathematical Society, vol. 347, no. 6, pp. 2075-2096, 1995.

[2] Y. Ling and G. Bao, "Some local Poincaré inequalities for the composition of the sharp maximal operator and the Green's operator," Computers \& Mathematics with Applications, vol. 63, no. 3, pp. 720-727, 2012.

[3] Z. Dai, Y. Xing, S. Ding, and Y. Wang, "Inequalities for the composition of Green's operator and the potential operator," Journal of Inequalities and Applications, vol. 2012, article 271, 13 pages, 2012.

[4] C. J. Neugebauer, "Inserting $A_{p}$-weights," Proceedings of the American Mathematical Society, vol. 87, no. 4, pp. 644-648, 1983.

[5] R. P. Agarwal, S. Ding, and C. Nolder, Inequalities for Differential Forms, Springer, New York, NY, USA, 2009.

[6] G. Bao, " $A_{r}(\lambda)$-weighted integral inequalities for $A$-harmonic tensors," Journal of Mathematical Analysis and Applications, vol. 247, no. 2, pp. 466-477, 2000. 
[7] Y. Xing, "A new weight class and Poincaré inequalities with the Radon measure," Journal of Inequalities and Applications, vol. 2012, article 32, 11 pages, 2012.

[8] H. Bi, "Weighted inequalities for potential operators on differential forms," Journal of Inequalities and Applications, vol. 2010, Article ID 713625, 13 pages, 2010.

[9] Y. Xing and S. Ding, "Norms of the composition of the maximal and projection operators," Nonlinear Analysis: Theory, Methods \& Applications, vol. 72, no. 12, pp. 4614-4624, 2010.

[10] T. Iwaniec and A. Lutoborski, "Integral estimates for null Lagrangians," Archive for Rational Mechanics and Analysis, vol. 125, no. 1, pp. 25-79, 1993.

[11] S. Ding, "Two-weight Caccioppoli inequalities for solutions of nonhomogeneous $A$-harmonic equations on Riemannian manifolds," Proceedings of the American Mathematical Society, vol. 132, no. 8, pp. 2367-2375, 2004. 


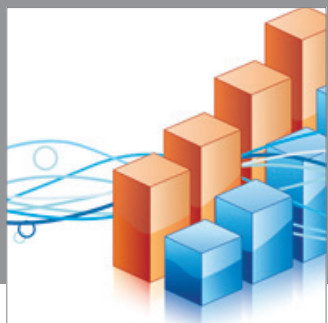

Advances in

Operations Research

mansans

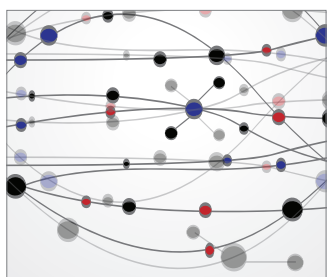

The Scientific World Journal
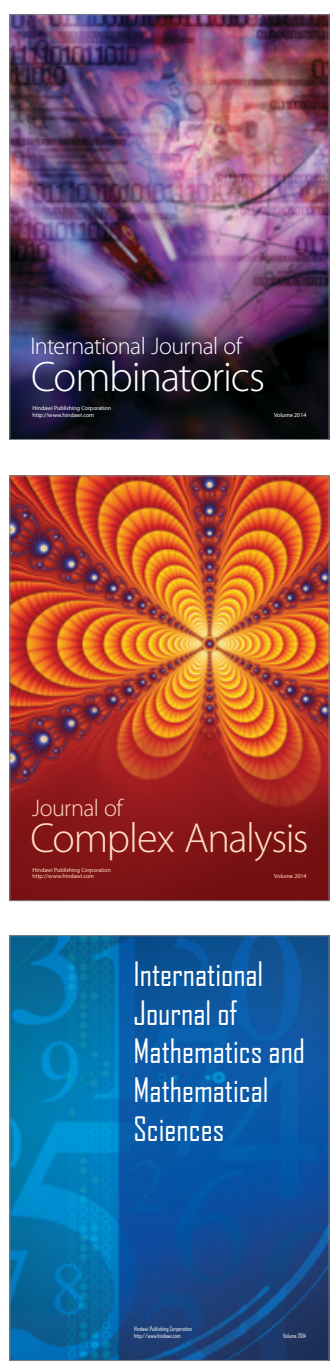
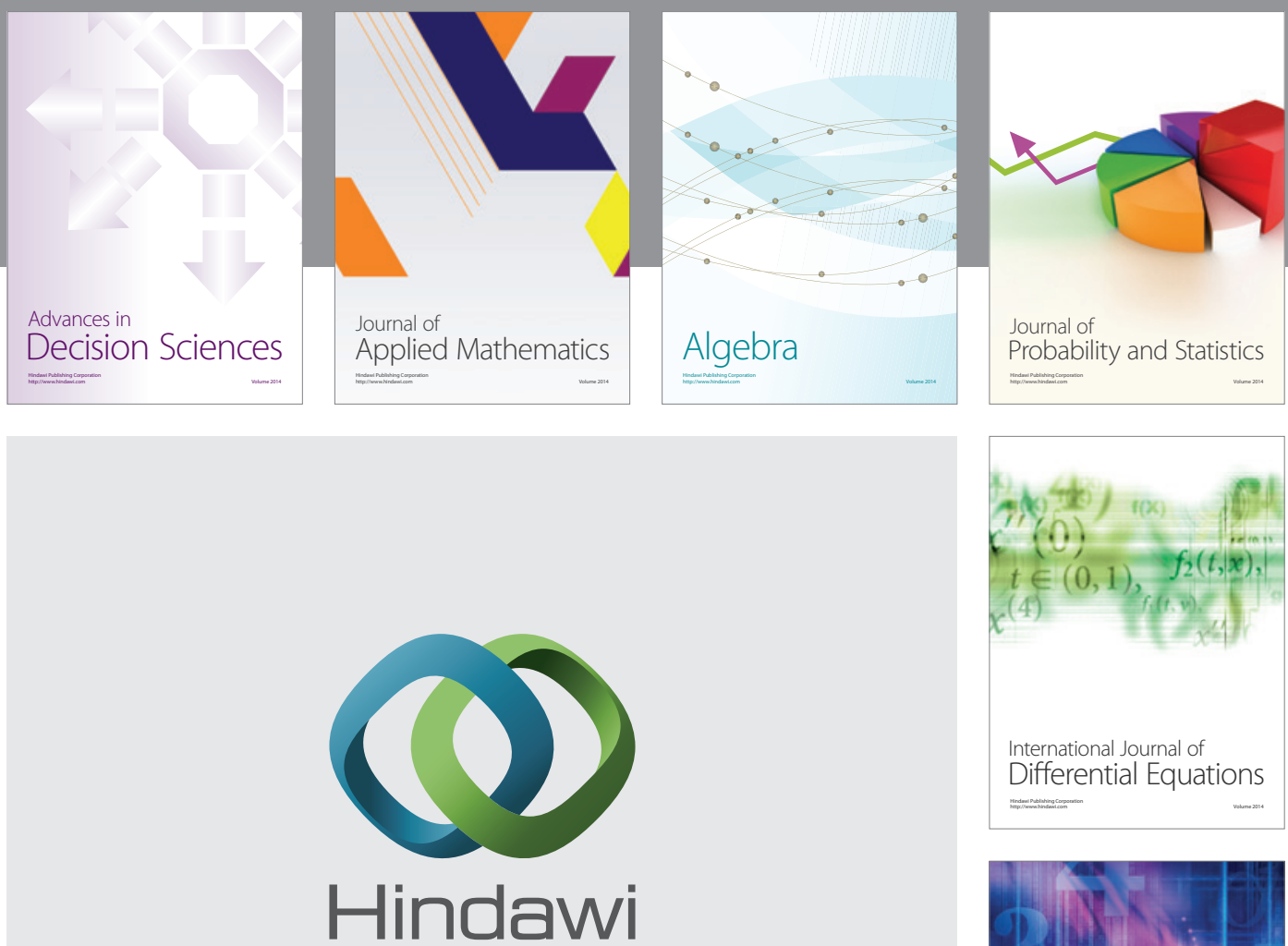

Submit your manuscripts at http://www.hindawi.com
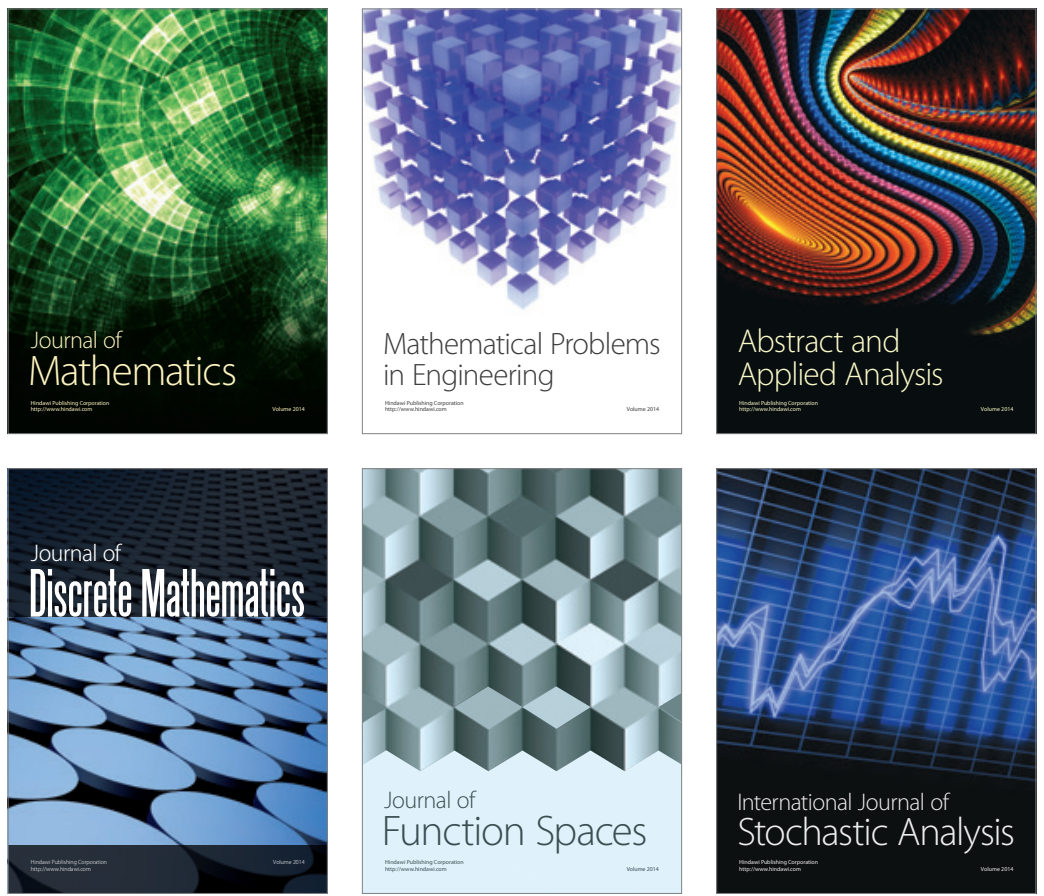

Journal of

Function Spaces

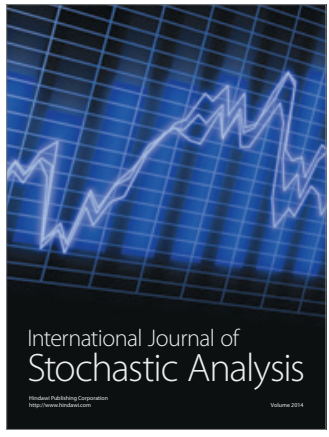

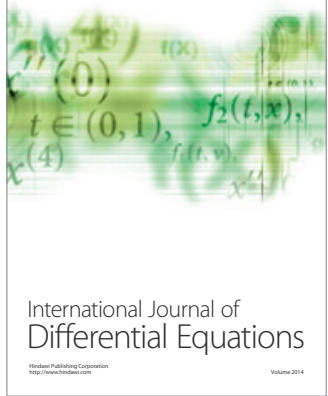
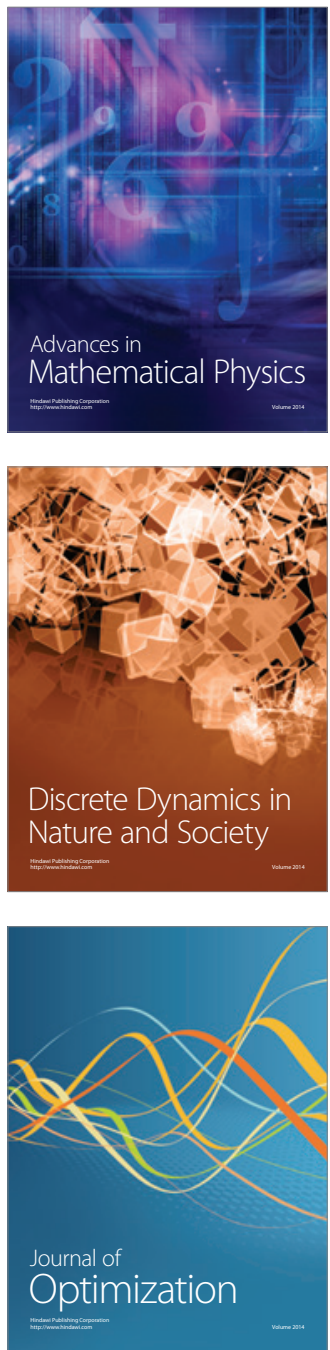